\title{
The Terminal Hosoya Polynomial of Some Families of Composite Graphs
}

\author{
Emeric Deutsch ${ }^{1}$ and Juan Alberto Rodríguez-Velázquez ${ }^{2}$ \\ ${ }^{1}$ Polytechnic Institute of New York University, Brooklyn, NY 11201, USA \\ ${ }^{2}$ Departament d'Enginyeria Informàtica i Matemàtiques, Universitat Rovira i Virgili, Avenida Països Catalans 26, \\ 43007 Tarragona, Spain
}

Correspondence should be addressed to Juan Alberto Rodríguez-Velázquez; juanalberto.rodriguez@urv.cat

Received 27 December 2013; Accepted 31 March 2014; Published 16 April 2014

Academic Editor: Jiang Zeng

Copyright (C) 2014 E. Deutsch and J. A. Rodríguez-Velázquez. This is an open access article distributed under the Creative Commons Attribution License, which permits unrestricted use, distribution, and reproduction in any medium, provided the original work is properly cited.

Let $G$ be a connected graph and let $\Omega(G)$ be the set of pendent vertices of $G$. The terminal Hosoya polynomial of $G$ is defined as $\operatorname{TH}(G, t):=\sum_{x, y \in \Omega(G): x \neq y} t^{d_{G}(x, y)}$, where $d_{G}(x, y)$ denotes the distance between the pendent vertices $x$ and $y$. In this note paper we obtain closed formulae for the terminal Hosoya polynomial of rooted product graphs and corona product graphs.

\section{Introduction}

Throughout this paper $G=(V, E)$ denotes a connected, simple, and finite graph with vertex set $V=V(G)$ and edge set $E=E(G)$. The distance $d_{G}(u, v)$ between two vertices $u$ and $v$ is the minimum of the lengths of paths between $u$ and $v$.

The Wiener index $W(G)$ of a graph $G$ with vertex set $\left\{v_{1}, v_{2}, \ldots, v_{n}\right\}$, defined as the sum of distances between all pairs of vertices of $G$,

$$
W(G):=\frac{1}{2} \sum_{i=1, j=1}^{n} d_{G}\left(v_{i}, v_{j}\right)
$$

is the first mathematical invariant reflecting the topological structure of a molecular graph.

This topological index has been extensively studied; for instance, a comprehensive survey on the direct calculation, applications, and the relation of the Wiener index of trees with other parameters of graphs can be found in [1]. Moreover, a list of 120 references of the main works on the Wiener index of graphs can be found in the referred survey.

The Hosoya polynomial of a graph was introduced in Hosoya's seminal paper [2] in 1988 and received a lot of attention afterwards. The polynomial was later independently introduced and considered by Sagan et al. [3] under the name Wiener polynomial of a graph. Both names are still used for the polynomial but the term Hosoya polynomial is nowadays used by the majority of researchers. The main advantage of the Hosoya polynomial is that it contains a wealth of information about distance based graph invariants. For instance, knowing the Hosoya polynomial of a graph, it is straightforward to determine the Wiener index of a graph as the first derivative of the polynomial at the point $t=1$. Cash [4] noticed that the hyper-Wiener index can be obtained from the Hosoya polynomial in a similar simple manner. A comprehensive survey on the main properties of the Hosoya polynomial, which includes a wide list of bibliographic references, can be found in Gutman et al. [5]. Also, Estrada et al. [6] studied several chemical applications of the Hosoya polynomial.

The Hosoya polynomial of a connected graph $G$ is defined as

$$
H(G, t):=\sum_{x, y \in V(G): x \neq y} t^{d_{G}(x, y)} .
$$

As we pointed out above, the Wiener index of a graph $G$ is determined as the first derivative of the polynomial $H(G, t)$ at $t=1$. 
The Hosoya polynomial has been obtained for trees, composite graphs, benzenoid graphs, tori, zig-zag openended nanotubes, certain graph decorations, armchair openended nanotubes, zigzag polyhex nanotorus, $\mathrm{TUC}_{4} \mathrm{C}_{8}(\mathrm{~S})$ nanotubes, pentachains, polyphenyl chains, the circumcoronene series, Fibonacci and Lucas cubes, Hanoi graphs, and so forth. See the references in [7].

The degree of a vertex $v$ in $G$ is the number of edges incident to it and is denoted by $\operatorname{deg}_{G}(v)$. If $\operatorname{deg}_{G}(v)=1$, then $v$ is called a pendent vertex of $G$. The set of pendent vertices of $G$ will be denoted by $\Omega(G)$.

Gutman et al. proposed the concept of terminal Wiener index, which is defined as the sum of distances between all pairs of pendent vertices of $G$ [8]. For detailed information on the terminal Wiener index the readers may refer to the recent papers [8-11] and the references cited therein.

By analogy to the definition of Hosoya polynomial, the terminal Hosoya polynomial was defined by Narayankar et al. $[12]$ as

$$
\mathrm{TH}(G, t):=\sum_{x, y \in \Omega(G): x \neq y} t^{d_{G}(x, y)},
$$

and recently Ramane et al. studied $\operatorname{TH}(G, t)$ for the case of line graphs [13]. As we can expect, the terminal Wiener index, denoted by TW( $G)$, is obtained from $\operatorname{TH}(G, t)$ as

$$
\operatorname{TW}(G)=\left.\frac{\partial(\mathrm{TH}(G, t))}{\partial t}\right|_{t=1} .
$$

Among the most known products of graphs, namely, Cartesian product, lexicographic product, strong product, rooted product, and corona product, the two latest ones are the only cases in which we can obtain connected graphs having pendent vertices. So, in this paper we obtain formulae for the terminal Hosoya polynomial of both types of graphs, rooted product graphs and corona product graphs.

The remaining definitions will be given the first time that the concept is found in the text.

\section{The Terminal Hosoya Polynomial of Rooted Product Graphs}

A rooted graph is a graph in which one vertex is labeled in a special way so as to distinguish it from the other vertices. The special vertex is called the root of the graph. Let $G$ be a labeled graph on $n$ vertices. Let $\mathscr{F}$ be a sequence of $n$ rooted graphs $G_{1}^{\prime}, G_{2}^{\prime}, \ldots, G_{n}^{\prime}$. The rooted product graph $G(\mathscr{F})$ is the graph obtained by identifying the root of $G_{i}^{\prime}$ with the $i$ th vertex of $G$ [14]. In this paper we consider a particular case of rooted product graphs where $\mathscr{F}$ consists of $n$ isomorphic rooted graphs [15]. More formally, assuming that $V(G)=$ $\left\{u_{1}, \ldots, u_{n}\right\}$ and that the root vertex of $G^{\prime}$ is $v$, we define the rooted product graph $G^{\circ}{ }_{v} G^{\prime}$ as the graph whose vertex set is $V\left(G \circ{ }_{v} G^{\prime}\right)=V(G) \times V\left(G^{\prime}\right)$ and whose edge set is

$$
\begin{aligned}
E\left(G \circ{ }_{v} G^{\prime}\right)= & \bigcup_{i=1}^{n}\left\{\left(u_{i}, b\right)\left(u_{i}, y\right): \text { by } \in E\left(G^{\prime}\right)\right\} \\
& \cup\left\{\left(u_{i}, v\right)\left(u_{j}, v\right): u_{i} u_{j} \in E(G)\right\} .
\end{aligned}
$$

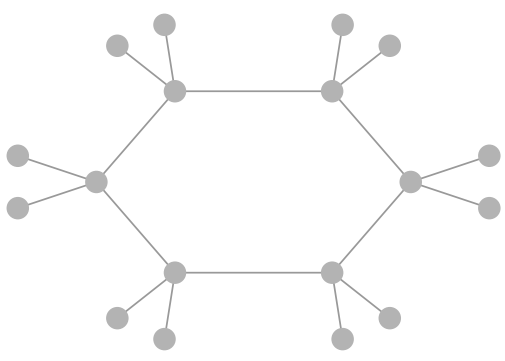

FIGURE 1: The graph $\mathrm{C}_{6}{ }^{\circ} \mathrm{P}_{3}$, where $\mathrm{C}_{6}$ is the cycle graph of order $6, \mathrm{P}_{3}$ is a path of order 3 , and $v$ is the central vertex of $\mathrm{P}_{3}$. This chemical graph that corresponds to the Cyclohexane (C6H12) is a naturally occurring chemical that is also produced synthetically and used as a solvent in numerous industries.

As an example of a chemical compound whose graph is obtained as a rooted product graph we consider the Cycloalkanes with a single ring, whose chemical formula is $\mathrm{C}_{n} \mathrm{H}_{2 n}$, and whose molecular graph can be expressed as $\mathrm{C}_{n} \circ{ }_{v} \mathrm{P}_{3}$, where $\mathrm{C}_{n}$ is the cycle graph of order $n, \mathrm{P}_{3}$ is a path of order 3 , and $v$ is the central vertex of $\mathrm{P}_{3}$ (see Figure 1).

To begin with, we need some additional notation. We define the terminal Hosoya polynomial with respect to $u$ as

$$
\mathrm{TH}_{u}(G, t):=\sum_{w \in \Omega(G)-\{u\}} t^{d_{G}(u, w)} .
$$

Theorem 1. Let $G$ be a connected graph of order $n \geq 2$ and let $G^{\prime}$ be a connected nontrivial graph. Then for any $v \in V\left(G^{\prime}\right)-$ $\Omega\left(G^{\prime}\right)$,

$$
T H\left(G \circ{ }_{v} G^{\prime}, t\right)=n \cdot T H\left(G^{\prime}, t\right)+H(G, t)\left(T H_{v}\left(G^{\prime}, t\right)\right)^{2} .
$$

Proof. Let $V(G)=\left\{u_{1}, u_{2}, \ldots, u_{n}\right\}$ and let $G_{i}^{\prime}$ be the $i$ th-"copy" of $G^{\prime}$ in $G^{\circ}{ }_{v} G^{\prime}$; that is, $G_{i}^{\prime}$ is the subgraph of $G^{\circ}{ }_{\nu} G^{\prime}$ generated by the set $\left\{u_{i}\right\} \times V\left(G^{\prime}\right)$, which is isomorphic to $G^{\prime}$. Then,

$$
\Omega\left(G \circ{ }_{v} G^{\prime}\right)=\bigcup_{i=1}^{n} \Omega\left(G_{i}^{\prime}\right) .
$$

So, if $\Omega\left(G^{\prime}\right)=\emptyset$, then $\Omega\left(G \circ{ }_{\nu} G^{\prime}\right)=\emptyset$ and, as a consequence, $\mathrm{TH}_{v}\left(G^{\prime}, t\right)=\mathrm{TH}\left(G^{\prime}, t\right)=\operatorname{TH}\left(G \circ{ }_{v} G^{\prime}, t\right)=0$. So, we assume that $\Omega\left(G^{\prime}\right) \neq \emptyset$.

Notice that for every pair $x, y$ of pendent vertices of $G^{\prime}$ and $u_{i} \in V(G)$ we have

$$
d_{G^{\circ} G^{\prime}}\left(\left(u_{i}, x\right),\left(u_{i}, y\right)\right)=d_{G_{i}^{\prime}}\left(\left(u_{i}, x\right),\left(u_{i}, y\right)\right)=d_{G^{\prime}}(x, y) .
$$

Also, for $u_{i}, u_{j} \in V(G), i \neq j$, we have

$$
\begin{aligned}
d_{G^{\circ} G^{\prime}}\left(\left(u_{i}, x\right),\left(u_{j}, y\right)\right)= & d_{G^{\prime}}(x, v)+d_{G}\left(u_{i}, u_{j}\right) \\
& +d_{G^{\prime}}(v, y) .
\end{aligned}
$$

Hence, $\mathrm{TH}\left(G \circ{ }_{v} G^{\prime}, t\right)$ splits as the sum of two polynomials $P(t)+Q(t)$, where $P(t)$ is obtained from pairs of pendent 
vertices in the same $G_{i}$ and $Q(t)$ is obtained from pairs $\left(u_{i}, x\right),\left(u_{j}, y\right)$, where $i \neq j$. On the one hand,

$$
P(t)=\sum_{i=1}^{n}\left(\sum_{x, y \in \Omega\left(G^{\prime}\right): x \neq y} t^{d_{G^{\prime}}(x, y)}\right)=n \cdot \mathrm{TH}\left(G^{\prime}, t\right),
$$

and, on the other hand,

$$
\begin{aligned}
Q(t)= & \sum_{u_{i}, u_{j} \in V(G): i \neq j} t^{d_{G}\left(u_{i}, u_{j}\right)}\left(\sum_{x \in \Omega\left(G^{\prime}\right)} t^{d_{G^{\prime}}(x, v)}\right) \\
& \times\left(\sum_{y \in \Omega\left(G^{\prime}\right)} t^{d_{G^{\prime}}(y, v)}\right) \\
= & \left(\operatorname{TH}_{v}\left(G^{\prime}, t\right)\right)^{2} \sum_{u_{i}, u_{j} \in V(G): i \neq j} t^{d_{G}\left(u_{i}, u_{j}\right)} \\
= & \left(\mathrm{TH}_{v}\left(G^{\prime}, t\right)\right)^{2} H(G, t) .
\end{aligned}
$$

Therefore, the result follows.

Given a vertex $v \in V\left(G^{\prime}\right)$, we define

$$
d_{T}(v):=\sum_{w \in \Omega\left(G^{\prime}\right)-\{v\}} d_{G^{\prime}}(v, w) .
$$

Notice that we can see $d_{T}(v)$ as the terminal Wiener index with respect to $v$; that is, $d_{T}(v)$ is the first derivative of the polynomial $\mathrm{TH}_{v}\left(G^{\prime}, t\right)$ at $t=1$. As a direct consequence of Theorem 1 we obtain the following result on the terminal Wiener index.

Corollary 2. Let $G^{\prime}$ be a connected nontrivial graph. Then for any $v \in V\left(G^{\prime}\right)-\Omega\left(G^{\prime}\right)$ and any connected graph $G$ of order $n \geq 2$,

$$
\begin{aligned}
T W\left(G \circ{ }_{v} G^{\prime}, t\right)= & n \cdot T W\left(G^{\prime}\right)+W(G)\left|\Omega\left(G^{\prime}\right)\right|^{2} \\
& +n(n-1)\left|\Omega\left(G^{\prime}\right)\right| d_{T}(v) .
\end{aligned}
$$

Now we consider the case of rooted product graphs $G^{\circ}{ }_{\nu} G^{\prime}$ where the root $v$ is a pendent vertex of $G^{\prime}$.

Theorem 3. Let $G$ be a connected graph of order $n \geq 2$ and let $G^{\prime}$ be a connected nontrivial graph. Then for any $v \in \Omega\left(G^{\prime}\right)$,

$$
\begin{aligned}
T H\left(G \circ_{v} G^{\prime}, t\right)= & n\left(T H\left(G^{\prime}, t\right)-T H_{v}\left(G^{\prime}, t\right)\right) \\
& +H(G, t)\left(T H_{v}\left(G^{\prime}, t\right)\right)^{2} .
\end{aligned}
$$

Proof. We proceed as in the proof of Theorem 1, with the only difference that in this case $P(t)$ is obtained as follows:

$$
\begin{aligned}
P(t) & =\sum_{i=1}^{n}\left(\sum_{x, y \in \Omega\left(G^{\prime}\right)-\{v\}: x \neq y} t^{d_{G^{\prime}}(x, y)}\right) \\
& =n\left(\sum_{x, y \in \Omega\left(G^{\prime}\right): x \neq y} t^{d_{G^{\prime}}(x, y)}-\sum_{x \in \Omega\left(G^{\prime}\right)-\{v\}} t^{d_{G^{\prime}}(x, v)}\right) \\
& =n\left(\mathrm{TH}\left(G^{\prime}, t\right)-\mathrm{TH}_{v}\left(G^{\prime}, t\right)\right) .
\end{aligned}
$$

Corollary 4. Let $G^{\prime}$ be a connected nontrivial graph. Then for any $v \in \Omega\left(G^{\prime}\right)$ and any connected graph $G$ of order $n \geq 2$, one has

$$
\begin{aligned}
T W\left(G{ }_{v} G^{\prime}, t\right)= & n\left(T W\left(G^{\prime}\right)-d_{T}(v)\right) \\
& +W(G)\left(\left|\Omega\left(G^{\prime}\right)\right|-1\right)^{2} \\
& +n(n-1)\left(\left|\Omega\left(G^{\prime}\right)\right|-1\right) d_{T}(v) .
\end{aligned}
$$

\section{The Terminal Hosoya Polynomial of Corona Graphs}

Let $G$ and $G^{\prime}$ be two graphs of order $n$ and $n^{\prime}$, respectively. The corona product $G \odot G^{\prime}$ is defined as the graph obtained from $G$ and $G^{\prime}$ by taking one copy of $G$ and $n$ copies of $G^{\prime}$ and joining by an edge each vertex from the $i$ th-copy of $G^{\prime}$ with the $i$ th-vertex of $G$ [16]. We will denote by $V=\left\{v_{1}, v_{2}, \ldots, v_{n}\right\}$ the set of vertices of $G$ and by $G_{i}^{\prime}=\left(V_{i}, E_{i}\right)$ the copy of $G^{\prime}$ such that $v_{i}$ is adjacent to $v$ for every $v \in V_{i}$. Thus, the vertex set of $G \odot G^{\prime}$ is $V \cup\left(\cup_{i=1}^{n^{\prime}} V_{i}\right)$ and, consequently, $G \odot G^{\prime}$ has order $n\left(n^{\prime}+1\right)$ and, if $G$ is connected, then $G \odot G^{\prime}$ is also connected and it has diameter $D\left(G \odot G^{\prime}\right)=D(G)+2$.

Notice that the corona graph $K_{1} \odot G^{\prime}$ is isomorphic to the join graph $K_{1}+G^{\prime}$. Now, if $G$ is a tree and $G^{\prime}=N_{r}$ is an empty graph (An empty graph on $r$ nodes consists of $r$ isolated nodes with no edges. Such graphs are sometimes also called edgeless graphs or null graphs), then the corona graph $G \odot N_{r}$ is a tree and the set of leaves (pendent vertices) of $G \odot N_{r}$ is $\cup_{i=1}^{n} V_{i}$. In particular, if $G=P$ is a path, then $P \odot N_{r}$ is a caterpillar graph and the path $P$, that is formed by the nonleaves of $P \odot N_{r}$, is known as the backbone of the caterpillar.

The formula for the Hosoya polynomial of corona graphs was previously obtained in [17]. In this section we obtain the formula for the terminal Hosoya polynomial of corona graphs $G \odot G^{\prime}$. Of course, $G \odot G^{\prime}$ has pendent vertices if and only if $G^{\prime}$ has isolated vertices.

Corollary 5. For any connected graph $G$ of order $n \geq 2$ and any graph $G^{\prime}$ having $r$ isolated vertices,

$$
T H\left(G \odot G^{\prime}, t\right)=n\left(\begin{array}{l}
r \\
2
\end{array}\right) t^{2}+r^{2} t^{2} H(G, t) .
$$

Proof. First of all, notice that $G \odot G^{\prime}=G^{\circ}{ }_{v}\left(\langle v\rangle+G^{\prime}\right)$, where $\langle v\rangle$ is the trivial graph composed by the vertex $v$. Now, since $\mathrm{TH}\left(\langle v\rangle+G^{\prime}, t\right)=\left(\begin{array}{c}r \\ 2\end{array}\right) t^{2}$ and $\mathrm{TH}_{v}\left(\langle v\rangle+G^{\prime}, t\right)=r t$, the result immediately follows from Theorem 1 .

Finally, Corollary 5 (and also Corollary 2) leads to the following formula for the terminal Wiener index of $G \odot G^{\prime}$.

Remark 6. For any connected graph $G$ of order $n \geq 2$ and any graph $G^{\prime}$ having $r$ isolated vertices,

$$
\operatorname{TW}\left(G \odot G^{\prime}\right)=n r(n r-1)+r^{2} W(G) .
$$




\section{Conflict of Interests}

The authors declare that there is no conflict of interests regarding the publication of this paper.

\section{References}

[1] A. A. Dobrynin, R. Entringer, and I. Gutman, "Wiener index of trees: theory and applications," Acta Applicandae Mathematicae. An International Survey Journal on Applying Mathematics and Mathematical Applications, vol. 66, no. 3, pp. 211-249, 2001.

[2] H. Hosoya, "On some counting polynomials in chemistry," Discrete Applied Mathematics, vol. 19, no. 1-3, pp. 239-257, 1988.

[3] B. E. Sagan, Y. Yeh, and P. Zhang, "The Wiener polynomial of a graph," International Journal of Quantum Chemistry, vol. 60, no. 5, pp. 959-969, 1996.

[4] G. G. Cash, "Relationship between the Hosoya polynomial and the hyper-Wiener index," Applied Mathematics Letters, vol. 15, no. 7, pp. 893-895, 2002.

[5] I. Gutman, Y. Zhang, M. Dehmer, and A. Ilic, "Altenburg, Wiener, and Hosoya polynomials," in Distance in Molecular Graphs-Theory, I. Gutman and B. Furtula, Eds., pp. 49-70, University of Kragujevac, Kragujevac, Serbia, 2012.

[6] E. Estrada, O. Ivanciuc, I. Gutman, A. Gutierrez, and L. Rodríguez, "Extended Wiener indices. A new set of descriptors for quantitative structure-property studies," New Journal of Chemistry, vol. 22, no. 8, pp. 819-822, 1998.

[7] E. Deutsch and S. Klavžar, "Computing the Hosoya polynomial of graphs from primary subgraphs," MATCH Communications in Mathematical and in Computer Chemistry, vol. 70, no. 2, pp. 627-644, 2013.

[8] I. Gutman, B. Furtula, and M. Petrović, "Terminal Wiener index," Journal of Mathematical Chemistry, vol. 46, no. 2, pp. 522-531, 2009.

[9] L. A. Székely, H. Wang, and T. Wu, "The sum of the distances between the leaves of a tree and the "semi-regular" property," Discrete Mathematics, vol. 311, no. 13, pp. 1197-1203, 2011.

[10] X. Deng and J. Zhang, "Equiseparability on terminal Wiener index," Applied Mathematics Letters, vol. 25, no. 3, pp. 580-585, 2012.

[11] Y.-H. Chen and X.-D. Zhang, "On Wiener and terminal Wiener indices of trees," MATCH Communications in Mathematical and in Computer Chemistry, vol. 70, no. 2, pp. 591-602, 2013.

[12] K. P. Narayankar, S. S. Shirkol, H. S. Ramane, and S. B. Lokesh, "Terminal Hosoya polynomial of thorn graphs," in International Conference on Discrete Mathematics (ICDM '13), Karnatak University, Dharwad, India, 2013.

[13] H. S. Ramane, A. B. Ganagi, K. P. Narayankar, and S. S. Shirkol, "Terminal Hosoya polynomial of line graphs," Journal of Discrete Mathematics, vol. 2013, Article ID 857908, 3 pages, 2013.

[14] C. D. Godsil and B. D. McKay, "A new graph product and its spectrum," Bulletin of the Australian Mathematical Society, vol. 18, no. 1, pp. 21-28, 1978.

[15] A. J. Schwenk, "Computing the characteristic polynomial of a graph," in Graphs and Combinatorics, R. Bari and F. Harary, Eds., pp. 153-172, Springer, Berlin, Germany, 1974.

[16] R. Frucht and F. Harary, "On the corona of two graphs," Aequationes Mathematicae, vol. 4, pp. 322-325, 1970.

[17] D. Stevanović, "Hosoya polynomial of composite graphs," Discrete Mathematics, vol. 235, no. 1-3, pp. 237-244, 2001. 


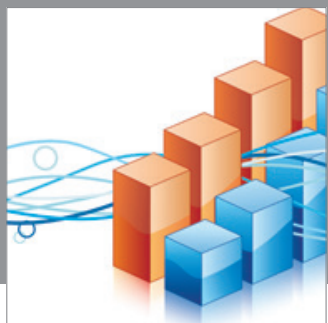

Advances in

Operations Research

mansans

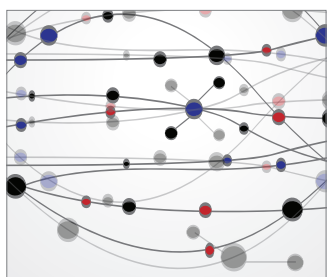

The Scientific World Journal
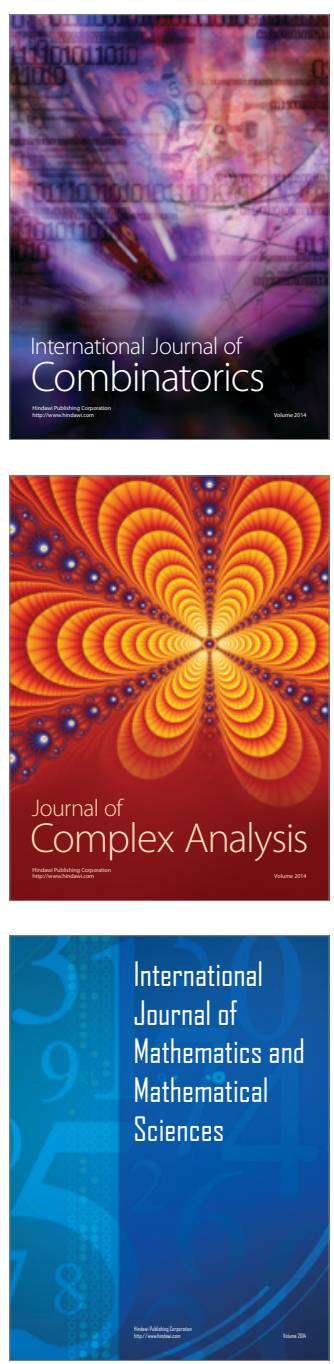
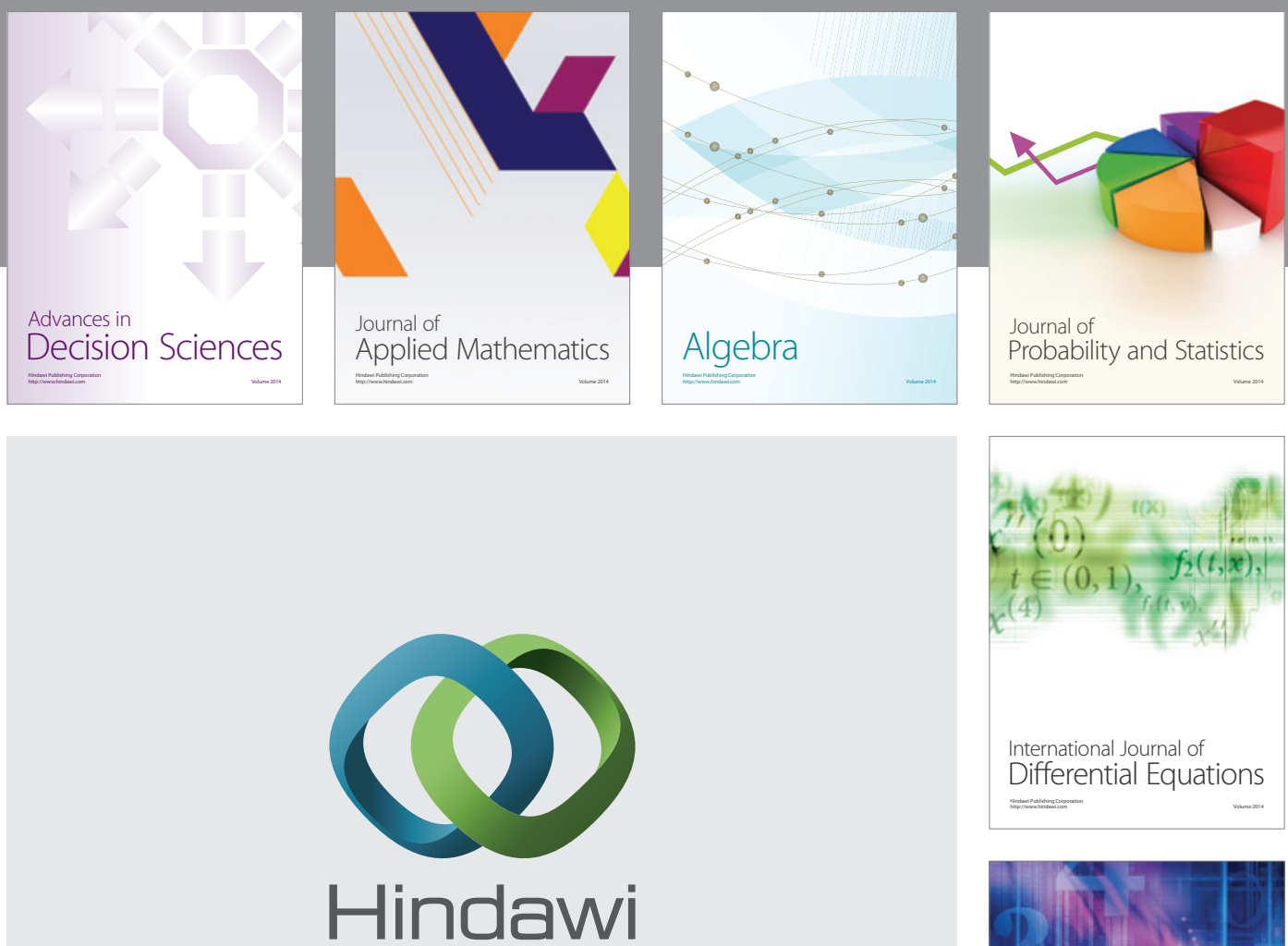

Submit your manuscripts at http://www.hindawi.com
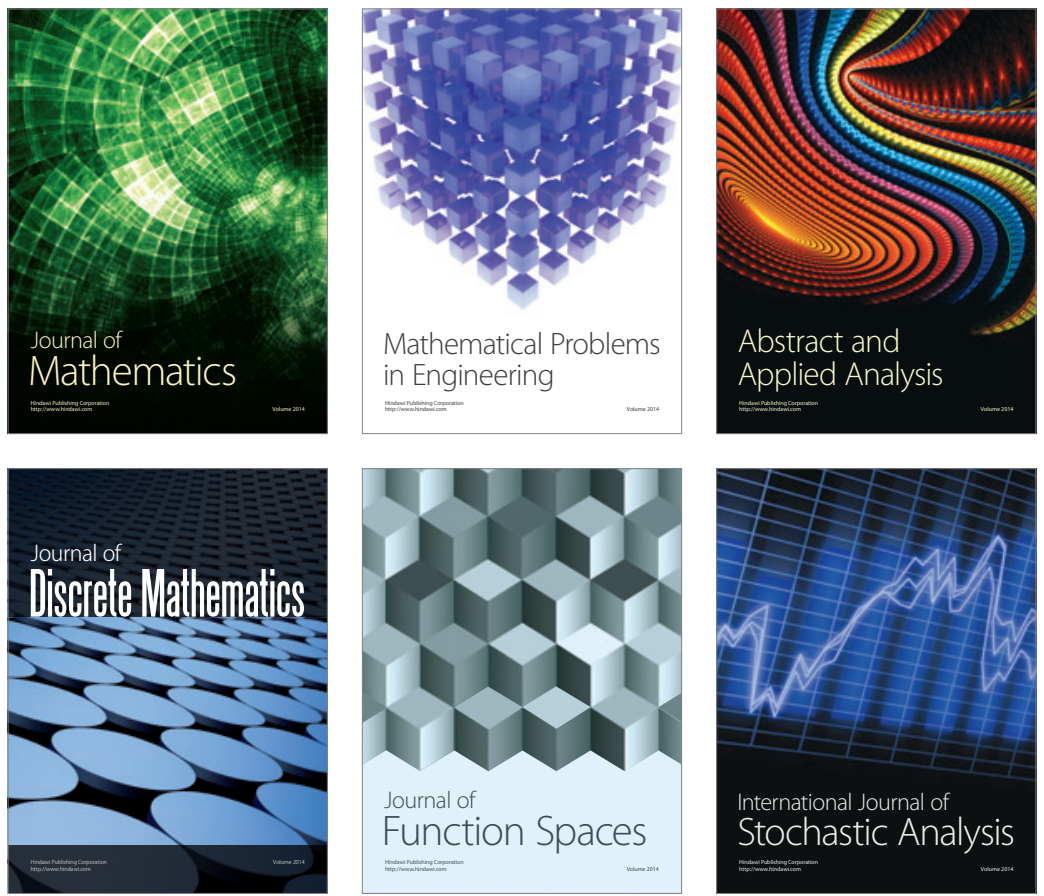

Journal of

Function Spaces

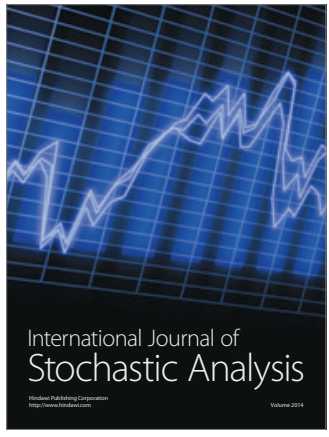

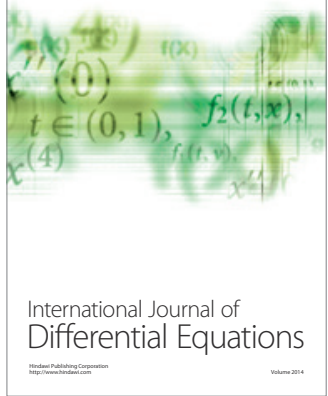
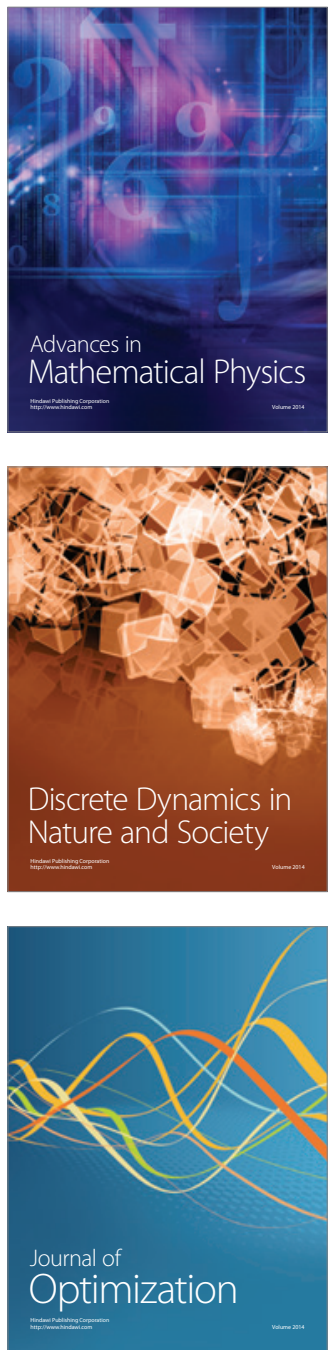